\title{
Minimal model analysis of insulin sensitivity and glucose-mediated glucose disposal in Type 1 (insulin-dependent) diabetic pancreas allograft recipients
}

\author{
K. Osei, D. A. Cottrell, M. L. Henry, R.J. Tesi, R. M. Ferguson and T.M. O'Dorisio \\ Department of Internal Medicine and Surgery, The Ohio State University Hospitals, Columbus, Ohio, USA
}

\begin{abstract}
Summary. Decreased insulin sensitivity and glucose-dependent glucose disposal (glucose effectiveness) have been demonstrated in poorly-controlled Type 1 (insulin-dependent) diabetic patients. We have therefore examined the effects of successful pancreas transplantation that results in long-term physiologic normoglycaemia as measured by insulin sensitivity index and glucose effectiveness in 14 Type 1 diabetic recipients (Group 1) using the Bergman minimal model method. Their results were compared with those of five non-diabetic patients with kidney transplant alone (Group 2) and 10 healthy control subjects (Group 3). Mean plasma glucose levels were indistinguishable in Group 1 when compared to Groups 2 and 3. However, mean basal plasma insulin levels were two- and eight-fold greater in Group $1(36 \pm 6 \mu \mathrm{U} / \mathrm{ml})$ than in Group $2(17 \pm 7 \mu \mathrm{U} / \mathrm{ml})$ and Group $3(4.5 \pm 0.6 \mu \mathrm{U} / \mathrm{ml})$, respectively. Following intra-
\end{abstract}

venous glucose $(t=0 \mathrm{~min})$ and tolbutamide $(t=20)$, peak incremental insulin levels were significantly $(p<0.001)$ greater in Group 1 vs Groups 2 and 3. Mean insulin sensitivity index was $65 \%$ and $50 \%$ lower in Group $1(2.89 \pm 0.45)$ and Group $2(4.11 \pm 1.30)$, respectively, when compared to Group $3\left(8.40 \pm 1.24 \times 10^{-1} \mathrm{~min}^{-1}(\mu \mathrm{U} / \mathrm{ml})^{-1}\right.$. In contrast, glucose effectiveness was similar in the three groups (Group 1, 2.48 \pm 0.26 ; Group 2, 2.05 \pm 0.21 ; and Group 3, $\left.2.10 \pm 0.17 \times 10^{-2} \cdot \mathrm{min}^{-1}\right)$. We conclude that, despite prednisone-induced insulin resistance, normal glucose tolerance is achieved by hyperinsulinaemia and normalisation of glucose-dependent glucose disposal following pancreas-kidney transplantation in Type 1 diabetic patients.

Key words: Pancreas transplantation, Type 1 (insulin-dependent) diabetes mellitus, insulin sensitivity.
Pancreas transplantation has improved the quality of life in Type 1 (insulin-dependent) diabetic recipients [1, 2]. When successful, pancreas transplantation achieves normal or near-normal glycaemia, however, the mechanisms of the latter remain uncertain. Hyperinsulinaemia, which is often a consequence of systemic venous insulin delivery, occurs in the face of normoglycaemia in some Type 1 diabetic pancreas allograft recipients with heterotopic, allograft location [3-6]. This hyperinsulinaemia indicates a state of severe insulin resistance in the diabetic allograft pancreas recipients. The insulin resistance has been ascribed partly to the prednisone therapy which is an important integral component of the currently used triple immunotherapy given to most organ recipients [3-8]. Secondly, hyperinsulinaemia per se could induce the insulin resistant state by down-regulation of the tissue insulin receptors [9]. Finally, poor glucose control prior to transplantation could also induce an insulin resistant state in Type 1 diabetic patients as a result of chronic glucose toxicity [10]. Thus, a clear understanding of the respective roles of hyperinsulinaemia and immunotherapy in the aetiology of insulin resistance in Type 1 allograft recipients is needed. Luzi et al. [11], using an elegant euglycaemic, hyperinsulinaemic technique have demonstrated improvement in insulin resistance in the skeletal muscles and normalization of hepatic glucose production following pancreas-kidney transplantation in Type 1 diabetic patients. The mechanism of the insulin resistance was ascribed to decreased insulin-mediated glucose disposal. However, whether glucose-dependent glucose disposal is decreased was not assessed in their study.

Previous investigators have shown that glucose-dependent glucose disposal is reduced in some Type 1 diabetic patients [12] but not others [13] in the face of decreased insulin-mediated glucose disposal using the euglycaemic, hyperinsulinaemic technique. The advent of the minimal model method described by Bergman and coworkers [14] has provided an opportunity for simultaneous determination of both insulin sensitivity $\left(\mathrm{S}_{1}\right)$ and glucose-dependent glucose disposal (glucose effectiveness, $\mathrm{S}_{\mathrm{G}}$ ). Recently, this methodology has been adapted for and validated in patients with insulin deficiency using 
Table 1. Clinical characteristics of subjects

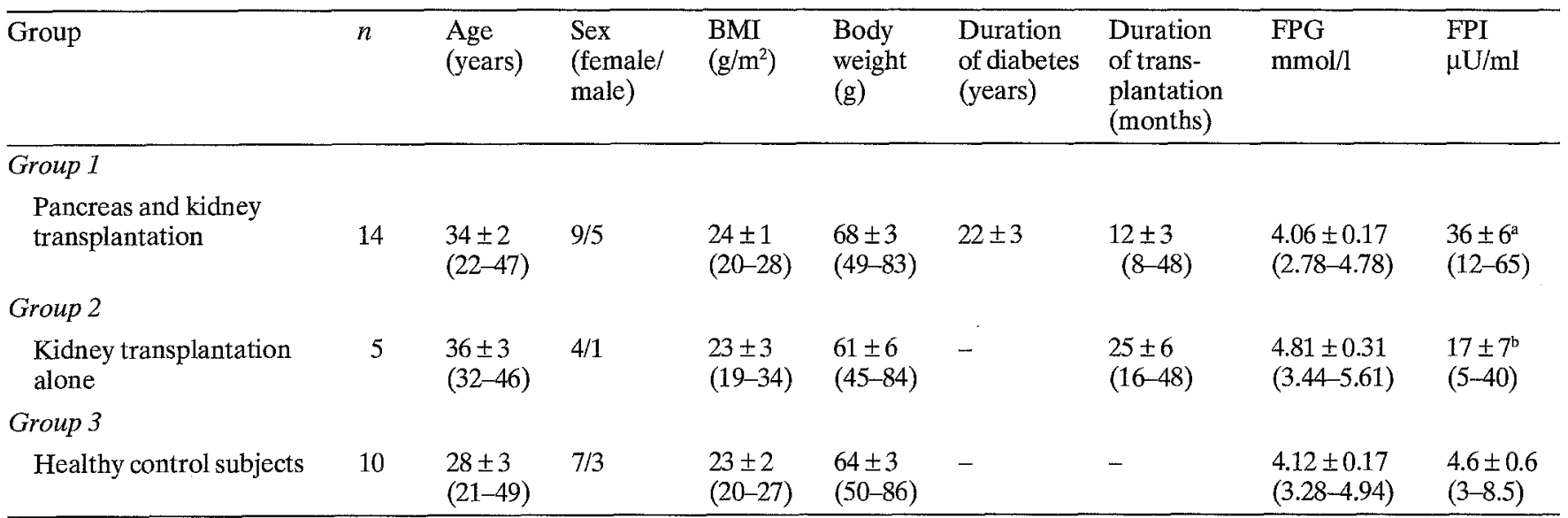

${ }^{\mathrm{a}} p<0.001 ;{ }^{\mathrm{b}} p<0.05$ vs healthy control subjects

Values are mean \pm SEM; numbers in parentheses represent range

FPG, Fasting plasma glucose; FPI, fasting plasma insulin

exogenous insulin administration resulting in peripheral rather than portal hyperinsulinaemia $[15,16]$. Thus, the objective of the present study was to employ the Bergman minimal model method [14] to investigate the mechanism(s) of the normal glucose tolerance in Type 1 diabetic patients who have undergone successful, whole cadaveric pancreas transplantation (resulting in peripheral hyperinsulinaemia and insulin-independence) and to compare the model-derived data with those of non-diabetic patients with kidney transplant alone treated with identical triple immunosuppressive agents and with the data of healthy control subjects.

\section{Subjects and methods}

Three study populations were recruited. Group 1 consisted of 14 Type 1 diabetic patients (nine females and five males) who underwent successful whole cadaveric pancreas and kidney transplantations as previously described [17] and were maintained on triple immunosuppressive therapy (cyclosporin, 250-500 mg/day, prednisone $10-15 \mathrm{mg} /$ day and azathioprine $125-150 \mathrm{mg}$ /day). All of the Type 1 diabetic allograft pancreas recipients had achieved long-term physiologic normoglycaemia and insulin-independence for a mean of 12 months (range 8-48 months). Thirteen of the diabetic patients underwent simultaneous pancreas and kidney transplantation. The remaining patient had previously received a kidney transplant approximately 1 year prior to the pancreas transplantation (sequential). The diabetic pancreas recipients were studied only if acute rejection episodes had not occurred during the 3 months prior to their visit to our research centre. Group 2 comprised five non-diabetic patients (four females and one male) who had received kidney transplantation alone for end-stage renal failure and were previously maintained on either haemo- or peritoneal dialysis. Following kidney transplantation, these patients were treated with a triple immunotherapy (prednisone 10-15 mg/day, cyclosporin 300-600 mg/day and azathioprine $75-150 \mathrm{mg} /$ day) similar to that of the Type 1 diabetic patients in Group 1. Group 3 consisted of ten healthy age-, sex- and weight-matched subjects (seven females and three males) who served as a control group. None of these subjects was taking any medication known to influence glucose tolerance or were participating in competitive or endurance sports. There was no family history of diabetes in the Group 3 subjects. Subjects in all three groups signed a written informed consent approved by the institutional re- view board after the risks entailed in the protocol had been thoroughly explained. Patients with renal, liver, heart and thyroid diseases were excluded. The clinical characteristics of the subjects are shown in Table 1.

\section{Study protocol}

All the studies were performed after a 10-12 h overnight fast at the Clinical Research Center of the Ohio State University Hospitals. Three days prior to the study, the subjects incorporated $200 \mathrm{~g}$ of carbohydrate in their regular weight-maintaining diet consisting of $50 \%$ carbohydrate, $40 \%$ fat and $10 \%$ protein in total energy content.

A standard oral glucose tolerance test was performed in all the subjects with $75 \mathrm{~g}$ glucose (Koladex Custom Laboratories, Baltimore, Md., USA). Using National Diabetes Data criteria, all the subjects were found to have normal glucose tolerance. The frequent sampling i. v. glucose tolerance (FSIGT) test was performed as previously described by Bergman and co-workers [14]. In brief, with the subject in the supine position, two i.v. lines were placed in both forearm veins. One was used for infusion of test substances and the other for blood sampling. Four blood samples were drawn at $t=-20,-10,-5$ and $0 \mathrm{~min}$. An i.v. glucose $(0.3 \mathrm{~g} / \mathrm{kg}, 50 \%$ dextrose) was infused over $1 \mathrm{~min}$ at $t=0 \mathrm{~min}$. At $t=20 \mathrm{~min}$, i.v. tolbutamide (Diagnostic Orinase, Upjohn Pharmaceuticals Company, Kalamazoo, Mich., USA) was infused over 1 min. Subjects with body mass index (BMI) less than $30 \mathrm{~kg} / \mathrm{m}^{2}$ received $300 \mathrm{mg}$ of tolbutamide while those with BMI greater than $30 \mathrm{~kg} / \mathrm{m}^{2}$ received $500 \mathrm{mg}$. Blood samples were drawn frequently at $t=0,2,3,5,6,8,10,12,14,16,18$, $20,22,25,30,35,40,45,50,60,70,80,90,100,110,120,150$ and $180 \mathrm{~min}$ for plasma glucose and insulin concentrations. Blood samples were immediately centrifuged at $4^{\circ} \mathrm{C}$, at a speed of $2,000 \times \mathrm{g}$ and $3,000 \mathrm{rev} / \mathrm{min}$. The plasma, stored at $-20^{\circ} \mathrm{C}$, was assayed for plasma insulin and glucose concentrations.

\section{Analytical methods}

Plasma glucose concentrations were measured by a glucose oxidase method using a glucose autoanalyser (Beckman Instruments, Fullerton, Calif, USA). Plasma insulin levels were determined by a standard double-antibody radioimmunoassay technique in our laboratory. The intra- and inter-assay coefficients of variation were $6 \%$ and 

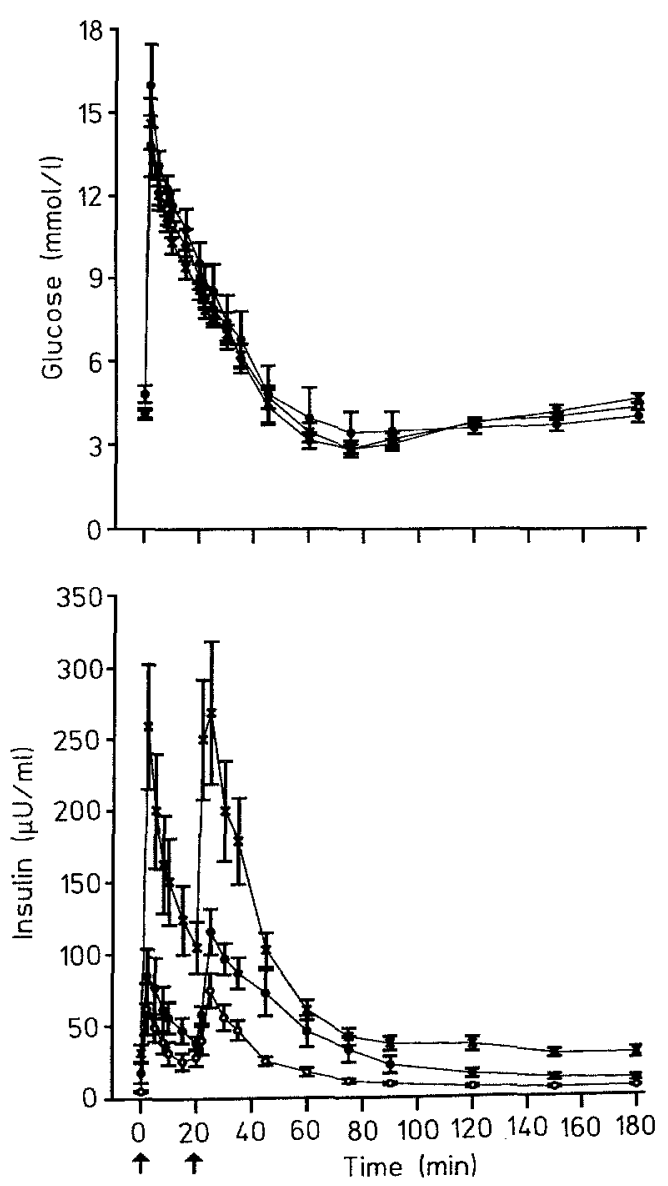

Fig.1. Plasma glucose (upper panel) and insulin (lower panel) given as mean \pm SEM before and after intravenous glucose $(t=0 \mathrm{~min})$ and tolbutamide $(t=20 \mathrm{~min})$ administration (arrows) in healthy control subjects $(\mathrm{O})$, patients with kidney transplant alone $(\bullet)$ and patients with both pancreas and kidney transplants (x)
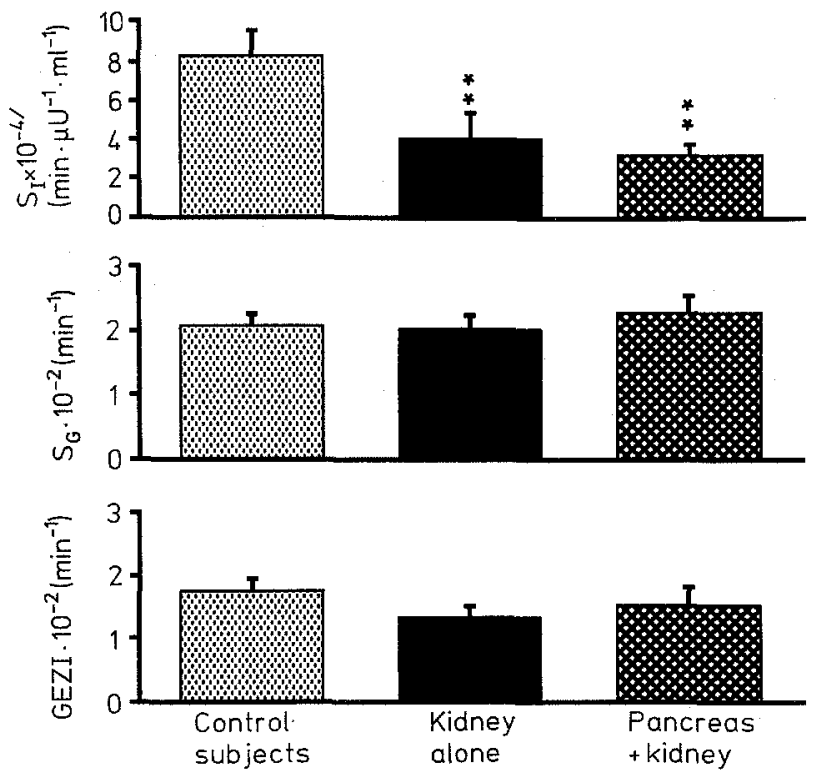

Fig. 2. Insulin sensitivity index $\left(S_{\mathrm{I}}\right)$, glucose effectiveness at basal insulin $\left(S_{G}\right)$ and glucose effectiveness at zero insulin (GEZI) (mean \pm SEM) shown for healthy control subjects ( ) , patients with kidney transplant alone ( $\mathbf{a})$ and patients with both pancreas and kidney transplants $(\$), * * p<0.001$
$10 \%$, respectively. The lower limit of the sensitivity of the insulin assay was $2.5 \mu \mathrm{U} / \mathrm{ml}$ of plasma.

\section{Statistical analysis}

Results are presented as mean \pm SEM unless otherwise stated. Glucose disappearance constant $\left(\mathbf{K}_{G}\right)$ after the i. v. glucose load was calculated from glucose concentrations between $t=8$ and $19 \mathrm{~min} . \mathrm{K}_{G}$ was taken as the negative slope of the linear regression equation between glucose concentrations (after natural log transformation) and time. Acute first phase insulin following $i$. v. glucose and tolbutamide was calculated as the sum of incremental plasma insulin levels between $t=0-5 \mathrm{~min}$ and $t=20-25 \mathrm{~min}$, respectively.

The $S_{\mathrm{Y}}$ and $\mathrm{S}_{\mathrm{G}}$ were calculated by the software program (MINIMOD) as previously described by Bergman and co-workers [14]. The minimal model parameters were computed based on glucose and insulin kinetics in each subject. Because $S_{G}$ reflects the glucose effectiveness at basal insulin concentrations, we calculated the true glucose effectiveness at the theoretical zero insulin concentrations (GEZI) as follows: (GEZI $=\mathrm{S}_{\mathrm{G}}-\mathrm{S}_{\mathrm{I}} \times \mathrm{BI}$ where BI represents the basal insulin concentration [18]. Also, since insulin sensitivity tends to be inversely related to basal insulin levels in the non-diabetic population, we calculated the disposition index (DI) which represents a product of $S_{\mathrm{I}}$ and Beta-cell insulin secretory parameter. DI was estimated for both the i.v. glucose and tolbutamide administration and designated as $\mathrm{DI}_{\text {gluc }}$ and $\mathrm{DI}_{\text {tolb }}$, respectively.

Non-parametric data were analysed using Mann-Whitney or chisquare methods. Student's $t$-test and where appropriate analysis of variance (ANOVA) for repeated measures were used for analyses of parametric data. Linear regression and correlation coefficients $(r)$ were calculated using the linear squares method. Probability value less than 0.05 was considered significantly different.

\section{Results}

As shown in Table 1, the mean age, sex ratio, body weight and body mass index were not significantly different between the immunosuppressant-treated patients (Groups 1 and 2) and the healthy control subjects (Group 3).

Mean fasting plasma glucose concentrations were not significantly different between the three groups (Group 1, $4.06 \pm 0.17 \mathrm{mmol} / \mathrm{l}$, Group 2, $4.81 \pm 0.31 \mathrm{mmol} / \mathrm{l}$ and Group 3, $4.12 \pm 0.17 \mathrm{mmol} / \mathrm{l})$. Following i.v. glucose load, the mean peak plasma glucose levels occurred at $2 \mathrm{~min}$ in all the groups. Mean plasma peak glucose levels were not significantly different between the groups (Group 1, $14.6 \pm 0.93 \mathrm{mmol} / \mathrm{l}, \quad$ Group $2, \quad 16 \pm 1.51 \mathrm{mmol} / \mathrm{l}$ and Group 3, $13.80 \pm 1.10 \mathrm{mmol} / 1$. Thereafter, the rates of glucose disappearance were similar (Fig.1, upper panel). Indeed, $\mathrm{K}_{\mathrm{G}}$ values were $1.79 \pm 0.20 \%$ per min for Group 1 , $2.11 \pm 0.6 \%$ per min for Group 2 and $2.0 \pm 0.30 \%$ per min for Group 3. The mean differences between the groups were not statistically significant.

Mean fasting plasma insulin concentrations were significantly greater in Group $1(36 \pm 6 \mu \mathrm{U} / \mathrm{ml})$ when compared to Group $2(17 \pm 7 \mu \mathrm{U} / \mathrm{ml}, p<0.02)$ and Group 3 $(4.5 \pm 0.6 \mu \mathrm{U} / \mathrm{ml}, p<0.001)$. Furthermore, after both i.v. glucose and tolbutamide administration, the peak and incremental plasma insulin responses were significantly greater in the pancreas transplant recipients (Group 1) when compared to the patients with kidney transplant 
alone (Group 2) and healthy control subjects (Group 3) as shown in Figure 1 (lower panel).

As shown in Figure 2 (upper panel), the mean insulin sensitivity index $\left(\mathrm{S}_{\mathrm{I}}\right)$ was significantly $(p<0.001)$ reduced by $65 \%$ in Group $1(2.89 \pm 0.45$ units $)$ and $50 \%$ in Group 2 (4.11 \pm 1.30 units) when compared to the healthy control subjects in Group $3\left[8.40 \pm 1.24 \times 10^{-4} \cdot \mathrm{min}^{-1}\right.$. $\left.(\mu \mathrm{U} / \mathrm{ml})^{-1}\right]$.

The mean disposition index (DI) after i.v. glucose was slightly but not significantly greater in Group 1 (1098 \pm 200 units) when compared to Group 2 (668 \pm 340 units) and Group 3 healthy control subjects $\left(662 \pm 153 \times 10^{-2} \cdot \mathrm{min}^{-1}\right)$. Mean $\mathrm{DI}_{\text {tolb }}$ values were similar to that of $\mathrm{DI}_{\text {gluc }}$ and mean differences were not statistically significant in the three groups.

Depicted in Figure 2 are the $S_{G}$ (middle panel) and GEZI (lower panel) data. Mean $S_{G}$ values were not significantly different between the three groups (Group 1, $2.48 \pm 0.26$, Group 2, $2.05 \pm 0.21$; and Group 3, $\left.2.10 \pm 0.17 \times 10^{-2} \cdot \mathrm{min}^{-1}\right)$. Similarly, the GEZI values were not different between the groups (Group 1, $1.56 \pm 0.41 ; \quad$ Group 2, $1.34 \pm 0.17$ and Group 3, $\left.1.77 \pm 0.18 \times 10^{-2} \cdot \mathrm{min}^{-1}\right)$.

The insulin sensitivity index $\left(\mathrm{S}_{\mathrm{l}}\right)$ correlated significantly but inversely with basal plasma insulin only in Group 1 $(r=-0.528, p<0.05)$ but not in Group $2(r=-0.177$, $p=\mathrm{NS})$ and Group $3(r=-0.482, p=\mathrm{NS})$, probably due to the clustering effects and smaller number of subjects in the latter two groups. Furthermore, no relationships existed between $S_{\mathrm{I}}$ or $\mathrm{S}_{\mathrm{G}}$ and age, body mass index, duration of functional allografts and dose of immunosuppressant drugs.

\section{Discussion}

Previous studies from our laboratory [3] and those of other investigators $[5,6]$ have demonstrated insulin resistance and hyperinsulinaemia in the face of normoglycaemia in Type 1 diabetic patients who receive whole cadaveric, heterotopic pancreas transplantation with exocrine drainage. Similarly, non-diabetic patients with kidney transplantation alone who receive triple immunosuppressive therapy including prednisone often manifest modest hyperinsulinaemia and a severe insulin resistant state $[3,5,6,19,20]$. The insulin resistance in both situations can result in recurrent glucose intolerance and diabetes in these immunosuppressed patients $[19,20]$. Most importantly, in pancreas transplant patients, the burden of insulin resistance, whatever its cause, could be potentially detrimental to the long-term function of the allograft pancreas. Thus, it is important to know whether the insulin resistance observed in diabetic and nondiabetic patients who receive allograft transplants have a common aetiologic mechanism.

We found that in Type 1 diabetic pancreas recipients, both fasting and post-stimulation plasma glucose concentrations are indistinguishable from that of kidney transplant patients (who receive identical triple immunotherapy) and healthy control subjects which is in agreement with several previous reports $[1,3-6]$. However, the nor- mal glucose concentration in the Type 1 diabetic pancreas recipients was achieved at the expense of insulin levels at least twice as high when compared to the kidney transplant patients in our study and eight times higher when compared to the healthy control subjects. Thus, it is tempting to infer that the insulin resistance appears to be more severe in the diabetic allograft recipients than the non-diabetic kidney patients. It is therefore possible that the mechanisms underlying the insulin resistant states may be different in both groups.

Using the euglycaemic clamp technique, Luzi et al. [11] have demonstrated similar degrees of insulin resistance in Type 1 diabetic pancreas recipients and patients with kidney transplants who were treated with comparable triple immunosuppressive agents. The insulin resistance has been ascribed predominantly to the prednisone therapy rather than cyclosporin and azathioprine components of the triple immunotherapy $[19,20]$. In the present study, we have quantitated the insulin sensitivity indices using Bergman's minimal model method. The $\mathrm{S}_{\mathrm{I}}$ values were reduced by $65 \%$ and $50 \%$ in the diabetic pancreatic allograft recipients and kidney transplant patients respectively, when compared to the healthy control subjects. It is important to note however, that the reduced $\mathrm{S}_{\mathrm{I}}$ in the diabetic pancreas recipients occurred in the face of two- to three-fold greater plasma insulin levels than the kidney transplant patients. Thus, the marked insulin resistance in the Type 1 diabetic pancreas recipients cannot be explained solely by the prednisone therapy as suggested by previous investigators. Moreover, the doses of prednisone in the two immunosuppressant groups were similar. We found that $S_{\mathbf{I}}$ correlated significantly but inversely with the basal plasma insulin levels only in the pancreatic transplant recipients but not in the kidney transplant patients and healthy control subjects. These correlation data should be interpreted cautiously since the lack of significant relationship between $S_{I}$ and basal insulin in the kidney transplant recipients could be due to the small number of subjects and a possible clustering effect. We, however, speculate that the marked systemic hyperinsulinaemia in the pancreas recipients could play a significant aetiologic role in the lower insulin sensitivity index following whole cadaveric, heterotopic, pancreas transplantation in Type 1 diabetic recipients [9]. It is noteworthy that hyperinsulinaemia could theoretically down-regulate the insulin receptors on insulin sensitive tissues. However, Saudek et al. [21] did not find any abnormality in insulin binding on erythrocytes of pancreas transplant recipients who manifested decreased insulin action and systemic hyperinsulinaemia.

Previous investigators have demonstrated that Type 1 diabetic patients with poor metabolic control manifest insulin resistance, and this is substantially improved by longterm intensive insulin therapy $[10,15]$. However, similar studies on $S_{G}$ have been very limited in immunosuppressed diabetic patients [15]. In this light, a recent report has demonstrated that both $S_{I}$ and $S_{G}$ are reduced in patients with Type 2 diabetes [22] and non-diabetic patients with Cushing's disease [23]. In contrast, Baron et al. [24] have shown that acute hydrocortisone infusion decreased only $S_{I}$ but not $S_{G}$ in humans. Thus, it is important to 
examine both parameters in patients who receive chronic corticosteroid therapy as an integral component of their immunosuppressive programme. We found, in our present study, a normal $\mathrm{S}_{\mathrm{G}}$ in the two transplant groups comparable to that of the healthy control subjects in Group 3. Similarly, the $K_{G}$, a surrogate of $S_{G}$, was also similar in the three groups. Thus, the immunosuppressive agents employed in our study do not appear to impair $S_{G}$ in allograft recipients.

In summary, the present study demonstrates that, in Type 1 diabetic allograft pancreas and kidney transplant recipients receiving similar immunosuppressive agents, $\mathrm{S}_{\mathrm{r}}$ is reduced by $65 \%$ and $50 \%$, respectively, when compared to healthy control subjects. Our data indicate that the insulin resistance in pancreas allograft recipients may be more severe when compared to that of kidney transplant patients and perhaps the marked systemic hyperinsulinaemia per se could be partly implicated. Thus, the normal $S_{G}$ in conjunction with hyperinsulinaemia may be responsible for the normal glucose concentrations observed in prednisone-induced, insulin-resistant patients receiving chronic immunosuppressive therapy.

Acknowledgements. We wish to express our gratitude to Ms. E.A. Robinson for her expert secretarial assistance, Mr. J. Spiropoulous for technical assistance, GCRC-RR34, National Institutes of Health, Bethesda, Maryland, USA, the Core Laboratories of The Ohio State University Hospitals, and the nursing staff of the Clinical Research Center. Dr. D. A. Cottrell is a recipient of Jack M. George, M.D.Fellowship Award and The Ohio State University Hospitals Fellowship Award.

\section{References}

1. Piehlmeier W, Bullinger M, Nusser J et al. (1991) Quality of life in type 1 (insulin-dependent) diabetic patients prior to and after pancreas and kidney transplantation in relation to organ function. Diabetologia 34 [Suppl 1]: S150-S159

2. Zehrer CL, Gross CR (1991) Quality of life of pancreas transplant recipients. Diabetologia 34 [Suppl 1] S138-S140

3. Osei K, Henry ML, O'Dorisio TM, Tesi RJ, Sommer BG, Ferguson RM (1990) Physiological and pharmacological stimulation of pancreatic islet hormone secretion in type 1 diabetic pancreas allograft recipients. Diabetes 39: 1235-1242

4. Östman J, Bolinder J, Gunnarson R et al. (1989) Effects of pancreas transplantation on metabolic and hormonal profiles in IDDM. Diabetes 38 [Suppl 1]: 88-93

5. Diem P, Abid M, Redmon JB, Sutherland DER, Robertson RP (1991) Systemic venous drainage of pancreas allografts as independent cause of hyperinsulinemia in type 1 diabetic recipients. Diabetes 39: 534-540

6. Robertson RP, Abid M, Sutherland DER, Diem P (1989) Glucose homeostasis and insulin secretion in human recipients of pancreas transplantation. Diabetes 38 [Suppl 1]: 97-98

7. Ekstrand A, Ahonen J, Grönhagen-Riska C, Groop L (1989) Mechanisms of insulin resistance after kidney transplantation. Transplantation 48: 563-568

8. Yale JF, Chamelian M, Courchesne S, Vigeant C (1988) Peripheral insulin resistance and decreased insulin secretion after cyclosporine A treatment. Transplant Proc 20: 985-988
9. Marangou AG, Weber KM, Boston RE et al. (1986) Metabolic consequences of prolonged hyperinsulinemia in humans: evidence of induction of insulin insensitivity. Diabetes 35: 13831389

10. Yki-Järvinen H, Helve E, Koivisto VA (1987) Hyperglycemia decreases glucose uptake in type 1 diabetes. Diabetes 36: 892896

11. Luzi L, Secchi A, Facchini F et al. (1990) Reduction of insulin resistance by combined kidney-pancreas transplantation in Type 1 (insulin-dependent) diabetic patients. Diabetologia 33: 549-556

12. Proietto J, Nankervis A, Aitken P, Caruso G, Alford F (1983) Glucose utilization in type 1 (insulin-dependent) diabetes: evidence for a defect not reversible by acute elevations of insulin. Diabetologia 25:331-335

13. Hansen IL, Cryer PE, Rizza RA (1985) Comparison of insulinmediated and glucose mediated glucose disposal in patients with insulin-dependent diabetes mellitus and in nondiabetic subjects. Diabetes 34: 751-755

14. Bergman RN, Prager R, Volund A, Olefsky NJ (1987) Equivalence of the insulin sensitivity index in man derived by the minimal model method and the euglycemic glucose clamp. J Clin Invest 79: 790-800

15. Finegood DT, Hramiak IM, Dupre J (1990) A modified protocol for estimation of insulin sensitivity with the minimal model of glucose kinetics in patients with insulin-dependent diabetes. J Clin Endocrinol Metab 70: 1538-1549

16. Ward GM, Weber KM, Walters IM et al. (1991) A modified minimal model analysis of insulin sensitivity and glucose-mediated glucose disposal in insulin-dependent diabetes. Metabolism 40: 4-9

17. Sollinger HW, Stratte RJ, Kalayoglu M, Pirsch JD, Belzer FO (1987) Pancreas transplantation with pancreatic cystostomy and quadruple immunosuppression. Surgery 102: 674-697

18. Kahn SE, Klaff LJ, Schwartz MW et al. (1990) Treatment with a somatostatin analog decreases pancreatic $\beta$-cell and whole body sensitivity to glucose. J Clin Endocrinol Metab 71: 994-1002

19. Gunnarson R, Arner P, Lundgren G, Magnusson G, Östman J, Groth CG (1979) Diabetes mellitus - a more common-than-believed complication of renal transplantation. Transplant Proc 11: $1280-1281$

20. Smith JL, Hunsicker LG, Yuh WTC, Wright FH, VanVoorhils L, Corry R (1989) Appearance of type 2 diabetes mellitus in type 1 diabetic recipients of pancreas allograft. Transplantation 47: 304-311

21. Saudek F, Pelikanova T, Bartos V et al. (1991) Insulin action and insulin binding following pancreas transplantation. Diabetologia 34 [Suppl 1]: S71-S75

22. Bergman RN (1989) Toward physiological understanding of glucose tolerance. Minimal model approach. Diabetes 38: 15121527

23. Page R, Boolell M, Kalfas A et al. (1991) Insulin secretion, insulin sensitivity and glucose-mediated glucose disposal in Cushing's disease: a minimal model analysis. Clin Endocrinol 35: 509517

24. Baron AD, Wallace P, Brechtel G (1987) In vivo regulation of non-insulin-mediated and insulin-mediated glucose uptake by cortisol. Diabetes 36: 1230-1237

Received: 17 December 1991

and in revised form: 25 March 1992

Dr. K.Osei

485 McCampbell Hall

1581 Dodd Drive

Columbus, OH 43210

USA 